\title{
Intravenöse Eisensubstitution bei chronischer Erkrankung - \\ bei wem, wann und wie?
}

\section{Intravenous Iron Substitution in Chronic Disease - in Whom, When and How?}

\author{
Autoren \\ Patrick Biggar ${ }^{1}$, Peter Langguth ${ }^{2}$, Pierre-Alexandre Krayenbühl' ${ }^{3}$, Vincent Brandenburg ${ }^{4}$
}

\section{(ㄷ) (1) $(9)$}

Institute

1 Klinik für Nephrologie, Klinikum Coburg GmbH

2 Institut für Pharmazie und Biochemie, Abteilung für Pharmazeutische Technologie und Biopharmazie,

J. G. Universität Mainz

3 Spital Linth, Schweiz

4 Klinik für Kardiologie und Nephrologie, Rhein-Maas Klinikum

\section{Schlüsselwörter}

Eisenmangelanämie, chronisch-entzündliche Erkrankung, Eisenpräparate, intravenöse Eisensubstitution

\author{
Key words \\ Bibliografie \\ DOI https://doi.org/10.1055/a-0810-8596 \\ Dtsch Med Wochenschr 2019; 144: 969-977 \\ (c) Georg Thieme Verlag KG, Stuttgart · New York \\ ISSN 0012-0472 \\ Korrespondenzadresse \\ Patrick Biggar \\ Klinikum Coburg GmbH \\ III. Medizinische Klinik - Nephrologie, \\ Ketschendorfer Straße 33, 96450 Coburg \\ patrick.biggar@regiomed-kliniken.de
}

iron deficiency anaemia, patients with chronic inflammatory diseases, iron preparations, intravenous iron substitution

\section{ZUSAMMENFASSUNG}

In den letzten Jahren konnten wesentliche Fortschritte in der Erkennung und Behandlung des Eisenmangels erzielt werden, deren Ergebnisse auch für viele Fachgebiete und insbesondere mit Patienten mit chronisch-entzündlichen Erkrankungen relevant sind. Allerdings wird in der täglichen Praxis ein Eisenmangel vielfach nicht identifiziert und konsequent therapiert. Ein Eisenmangel kann - auch bevor eine Anämie auftritt - die Lebensqualität einschränken und den Verlauf der Grunderkrankung beeinflussen. Bei Patienten mit chronischen Erkrankungen sollte der Eisenstatus regelmäßig erfasst werden. Gerade bei Patienten mit chronisch-entzündlichen Erkrankungen sind die derzeit verfügbaren oralen Eisenpräparate ineffizient, weil die Verträglichkeit eingeschränkt ist und die enterale Eisenaufnahme durch Inflammation eingeschränkt sein kann. Deshalb empfehlen verschiedene Leitlinien hier eine intravenöse Eisensubstitution.

\section{ABSTRACT}

In recent years, considerable progress has been made in the detection and treatment of iron deficiency. The results are also relevant for many specialist areas and, in particular, for patients with chronic inflammatory diseases. In daily practice, iron deficiency is often neither identified nor consistently treated.

An iron deficiency can - even before anaemia occurs - reduce the quality of life and influence the course of the underlying disease. In patients with chronic diseases, the iron status should be monitored regularly. Especially, the currently available oral iron preparations for these patients are inefficient, because of the limitated tolerability and, furthermore, because of restricted enteral iron uptake due to inflammation. For this reason, various guidelines recommend intravenous iron substitution.

\section{Einleitung}

Eisenmangelanämie ist ein weltweites Gesundheitsproblem. Besonders Patienten mit chronischer Nieren- und Herzinsuffizienz, mit chronisch-entzündlichen Darmerkrankungen sowie Malignomen haben ein erhöhtes Risiko für ein Eisendefizit. Der Eisenmangel schränkt die Lebensqualität ein und er kann auch den Verlauf der Grunderkrankung verschlechtern [1].
In unkomplizierten Fällen können orale Eisenpräparate eingesetzt werden. Jedoch empfehlen internationale Leitlinien intravenöses (i. v.) Eisen besonders bei hohem Eisenbedarf, da es effektiver ist und besser toleriert wird als orales Eisen [2]. Derzeit stehen mehrere i.v. Eisenpräparate zur Verfügung. Auch wenn das Risiko einer anaphylaktischen Reaktion bei modernen Eisenpräparaten geringer ist als bei den in Deutschland nicht mehr 
erhältlichen, hochmolekularen Dextranpräparaten [3], werden Sicherheitsvorkehrungen peri-infusionem empfohlen [4].

\section{Eisenmangelsymptome}

Ein Eisenmangel resultiert, wenn ein Missverhältnis zwischen Eisenaufnahme, Verbrauch und Verlust besteht (siehe: Ursachen). Die Symptome eines Eisenmangels sind unspezifisch: Leistungsminderung, Schwindel, Schleimhautveränderungen, Tinnitus oder neurologische Symptome wie das restless-legs-Syndrom. Sie entstehen u. a. durch die Sauerstoffunterversorgung sowie durch die Beeinträchtigung Eisen-abhängiger Enzyme wie beispielsweise Cytochrom-C-Proteine der Atmungskette, die für die mitochondriale Energiegewinnung essenziell sind. Ein Eisenmangel kann sich pathophysiologisch bereits vor Entstehung einer Anämie bemerkbar machen [5].

Vielfältige Ursachen für einen Eisenmangel:

- ein kontinuierlicher Blutverlust, beispielsweise durch gastrointestinale Blutungen, Hypermenorrhoe, regelmäßige Blutabnahmen und Operationen

- ein gesteigerter Eisenbedarf bei einer Therapie mit Erythropoetin-stimulierenden Agenzien (ESAs)

- verminderte enterale Eisenaufnahme, entweder durch die Erkrankung selbst oder durch Medikamente wie Protonenpumpenhemmer oder Phosphatbinder

- Mangelernährung, Diätvorschriften oder -wünsche

Die klinische Diagnose des Eisenmangels wird erschwert, da Symptome der Grunderkrankung zugerechnet werden können. So ergab eine deutsche Erhebung, dass bei $40 \%$ der Patienten mit Herzinsuffizienz ein bis dahin unbekannter Eisenmangel vorgelegen hatte [6]. In einer ebenfalls deutschen Kohorte mit Patienten mit chronisch-entzündlichen Darmerkrankungen (CED) erhielt die Hälfte trotz diagnostizierter Eisenmangelanämie keine Eisensubstitution [7]. Ein Eisenmangel ist nicht nur mit einer eingeschränkten Lebensqualität, sondern auch mit einem schlechteren Outcome der Grunderkrankung assoziiert. Hierzu liegen größere kardiologische, nephrologische und onkologische Studien vor [8]. Somit erscheint bei diversen Erkrankungen eine Eisensubstitution bereits vor Entwicklung einer Anämie sinnvoll [9].

\section{Physiologische Eisenregulation}

Bei Gesunden beträgt der Gesamteisengehalt des Körpers etwa $4 \mathrm{~g}$. Der Eisenverlust liegt bei ca. 1,5 mg täglich. Er wird durch Aufnahme von Eisen mit der Nahrung kompensiert; dabei werden nur bis $10 \%$ des oral zugeführten Eisens resorbiert. Bei Eisenmangel kann der Anteil physiologisch auf maximal $5 \mathrm{mg}$ Eisen pro Tag verdreifacht werden [10]. Eine zentrale Rolle in der Eisenregulation spielt das Hormon Hepcidin. Es wird bei ausreichender Eisenversorgung ausgeschüttet und inhibiert sowohl die Aufnahme von Eisen aus der Nahrung als auch die Verfügbarkeit im Körper, indem es die Ferroportin-Expression auf den Zelloberflächen, z. B. auf der basolateralen Membran von duodenalen Enterozyten und auf der Oberfläche von Hepatocyten sowie von Makrophagen, down-reguliert [11].

\section{Chronische Krankheiten und Eisenmangel}

Viele chronisch Erkrankte weisen einen Eisenmangel auf. Dieser beruht wesentlich auf einer systemischen Inflammation mit einer vermehrten Ausschüttung von Hepcidin mit Blockade der Eisenverfügbarkeit im Körper.

Zwischen 24 und $85 \%$ aller Patienten mit chronischen Nierenerkrankungen und bis zu $50 \%$ der Patienten mit Herzinsuffizienz weisen in Abhängigkeit vom Erkrankungsstadium einen Eisenmangel auf [12]. Bei CED sind es 13 bis $90 \%$ [1]. Eine Anämie tritt bei rund 2/3 aller Krebspatienten auf, ein absoluter Eisenmangel zwischen 7 und $46 \%$ [13].

\section{Eisenstatus gezielt überprüfen}

Ein isolierter Serumeisenwert ist ungenügend verwertbar. Mikrozytäre, hypochrome Erythrozyten können im Blutbild Hinweise auf einen Eisenmangel geben, wobei die Auswertung von 3104 nichtschwangeren Frauen im Rahmen der amerikanischen NHANESUntersuchung in den Jahren 1999 - 2006 bei einer angesetzten Spezifität von $80 \%$ eine Sensitivität von nur $61 \%$ des mittleren zellulären Volumens (MCV) ergab [14]. Die vorrangigen Eisenparameter im Alltag sind Serumferritin und Transferritinsättigung (TSAT).

Das Serumferritin korreliert mit dem Körpereisenbestand mit Normwerten 30 - $100 \mathrm{ng} / \mathrm{ml}$. Ferritin wird überwiegend von Makrophagen sezerniert, weniger von Hepatozyten. Zwar beweist ein erniedrigtes Ferritin einen Eisenmangel, jedoch können sich hinter einem normalen oder erhöhten Wert verschiedene klinische Zustände verbergen, da Ferritin als Akutphasenprotein bei Entzündungen mit ansteigt. Ferritinwerte über $800 \mathrm{ng} / \mathrm{ml}$ können, müssen jedoch nicht auf eine Eisenüberladung hindeuten [15]. Das Serumferritin kann erhöht sein, obwohl im Knochenmark kein Eisen nachzuweisen ist.

Unabhängiger vom Entzündungsstatus ist die TransferrinSättigung (TSAT). Eine TSAT < 20 \% spricht für einen Eisenmangel. Die TSAT spiegelt den funktionellen Eisenstatus besser wider als Ferritin. Im Vergleich zum Serumferritin gilt die TSAT prinzipiell als der zuverlässigere Parameter, um ein Ansprechen des Blutbildes auf Eisengabe vorherzusagen [16].

\section{Eisenbedarf abschätzen}

Die Ganzoni-Formel ermöglicht anhand des Hämoglobins und des Körpergewichts zuzüglich einer Eisenreserve die Schätzung des Eisenbedarfs [17] ( $\triangleright$ Tab. 1). Zusätzlich zum Grundbedarf benötigen nicht-dialysepflichtige Nierenerkrankte etwa zusätzlich rund $0,4 \mathrm{~g}$ Eisen pro Jahr, dialysepflichtige Patienten zwischen 1 und $2 \mathrm{~g}$. Bei Patienten mit entzündlichen Darmerkrankungen sowie mit onkologischen Erkrankungen wurde der zusätzliche Eisenbedarf auf $>1 \mathrm{~g}$ pro jahr geschätzt. 
- Tab. 1 Eisenbedarf nach Ganzoni 1970. Gesamteisendefizit = Gewicht $\{\mathrm{kg}\} \times($ Ziel Hb - aktuelles $\mathrm{Hb})\{\mathrm{g} / \mathrm{l}\} \times 2,4$ + Eisenreserve $\{\mathrm{mg}\}(=500 \mathrm{mg})$. Vereinfachte Schätzung:

\begin{tabular}{|l|l|l|}
\hline Hb-Wert & $\begin{array}{l}\text { Patienten mit einem } \\
\text { Körpergewicht von } \\
\mathbf{5 0 ~ b i s < 7 0 ~ k g}\end{array}$ & $\begin{array}{l}\text { Patienten mit einem } \\
\text { Körpergewicht von } \\
\geq \mathbf{7 0 ~} \mathbf{~ g g}\end{array}$ \\
\hline $\begin{array}{l}>10 \mathrm{~g} / \mathrm{dl} \\
(>6,2 \mathrm{mmol} / \mathrm{l})\end{array}$ & $1000 \mathrm{mg}$ & $1500 \mathrm{mg}$ \\
\hline $\begin{array}{l}<10 \mathrm{~g} / \mathrm{dl} \\
(<6,2 \mathrm{mmol} / \mathrm{l})\end{array}$ & $1500 \mathrm{mg}$ & $2000 \mathrm{mg}$ \\
\hline
\end{tabular}

\section{Behandlung mit Eisensubstitution}

Orale Eisenpräparate haben Nachteile im Bereich der Verträglichkeit und Effektivität. Eine Metaanalyse von randomisierten, kontrollierten Studien von Eisensulfat hatte signifikante gastrointestinale Nebenwirkungen bei $>70 \%$ der 6831 Probanden ergeben [18].

Bei einer Eisenresorption von 5 mg pro Tag bei Gesunden kann eine Therapiedauer von einigen Monaten notwendig sein. Jedoch führt die inflammatorische Suppression der Eisenresorption bei chronisch Kranken zu einer verminderten Effektivität der oralen Therapie.

Bei intravenöser Zufuhr werden bei deutlich weniger gastrointestinalen Nebenwirkungen [19] die inflammatorische Inhibition der enteralen Eisenresorption und andere Malassimilationsprobleme umgangen. Gleichzeitig steigert eine überwachte Infusion die Compliance. Durch intravenöse Applikation lassen sich mittels Eisencarboxymaltose bis zu $1000 \mathrm{mg}$ und mittels Derisomaltose (alternative chemische Bezeichnung: Eisenisomaltosid) bis zu max. 20 mg/kg Körpergewicht Eisen auf einmal verabreichen. Ein weiteres Hochdosispräparat, Ferumoxytol, wird aktuell in Europa nicht vertrieben. Der Metabolismus der Eisenverwertung dieser Substanzen ist vergleichbar mit der Erythrophagozytose durch Makrophagen.

Verschiedene Fachleitlinien berücksichtigen die Eigenschaften der oralen und intravenösen Eisensubstitution (Zielwerte in

- Tab. 2):

- KDIGO (Kidney Disease - Improving Global Outcomes): nichtdialysepflichtige chronisch Nierenerkrankte können sowohl orales als auch intravenöses Eisen erhalten. Falls eine Hämoglobinanhebung unter oralem Eisen innerhalb von 1 bis 3 Monaten nicht erreicht wird, soll eine intravenöse Applikation in Erwägung gezogen werden. Dialysepflichtige Patienten werden primär mit intravenösem Eisen behandelt bevor ESAs zum Einsatz kommen [2].

- Die Leitlinien der European Society of Cardiology (ESC) sehen vor, dass Herzinsuffizienz-Patienten mit Eisenmangel eine Therapie mit intravenösem Eisen (explizit Eisencarboxymaltose) erhalten [20].

- Die europäischen Empfehlungen zu CED sehen vor, dass orales Eisen bei Patienten mit milder Anämie zum Einsatz kommen kann, solange die Erkrankung nicht aktiv ist. Hingegen sollten Patienten mit einer klinisch aktiven Erkrankung, einem
- Tab. 2 Eisenparameter Mindestwerte.

\begin{tabular}{|c|c|c|}
\hline Grunderkrankung & $\begin{array}{l}\text { Serum-Ferritin } \\
\text { (ng/ml) }\end{array}$ & $\begin{array}{l}\text { Transferrin- } \\
\text { Sättigung }\end{array}$ \\
\hline ohne Grunderkrankung & $>30$ & $>20$ \\
\hline \multicolumn{3}{|l|}{$\begin{array}{l}\text { chronische Nieren- } \\
\text { erkrankung }\end{array}$} \\
\hline - nicht dialysepflichtig & $>200$ & $>25$ \\
\hline - dialysepflichtig & $>300$ & $>25$ \\
\hline $\begin{array}{l}\text { chronische } \\
\text { Herzinsuffizienz }^{1}\end{array}$ & $>100$ & $\begin{array}{l}>20 \text { (bei Ferritin } \\
100-299 \mathrm{ng} / \mathrm{ml} \text { ) }\end{array}$ \\
\hline \multicolumn{3}{|l|}{$\begin{array}{l}\text { chronisch entzündliche } \\
\text { Darmerkrankung }\end{array}$} \\
\hline - ohne Krankheitsaktivität & $>30$ & $>20$ \\
\hline - mit Krankheitsaktivität & $>100$ & $>20$ \\
\hline \multicolumn{3}{|l|}{$\begin{array}{l}\text { karzinom- und Chemothe- } \\
\text { rapiebedingte Anämie (Hb } \\
\leq 11 \mathrm{~g} / \mathrm{dl} \text { ) }\end{array}$} \\
\hline - ohne Entzündung & $\geq 100$ & $\geq 20$ \\
\hline - mit Entzündung 3 & $\geq 100$ & $\geq 20$ \\
\hline \multicolumn{3}{|c|}{$\begin{array}{l}1 \text { Studienkriterien Ponikowski [20] und Anker [34]. } \\
2 \text { Dignass [21]. } \\
3 \text { Aapro [22]. }\end{array}$} \\
\hline
\end{tabular}

Hb-Wert unter $10 \mathrm{~g} / \mathrm{dl}$, einem ESA-Bedarf sowie Unverträglichkeit gegenüber oralem Eisen eine intravenöse Eisentherapie erhalten [21].

- Die 2018 Leitlinien der europäischen Gesellschaft für medizinische Onkologie (ESMO) empfehlen intravenöses Eisen bei $\mathrm{Hb} \leq 11 \mathrm{~g} / \mathrm{dL}$ und einem Serumferritin $<100 \mathrm{ng} / \mathrm{mL}$, ferner im Einzelfall bei einem funktionellen Eisenmangel (TSAT $<20 \%$ und Serumferritin $>100 \mathrm{ng} / \mathrm{mL}$ ) [22].

\section{Historische Entwicklung und chemische Strukturen der i. v. Eisenpräparate}

Die zeitliche Entwicklung der Substanzen wird in $\mathbf{A}$ Abb. $\mathbf{1}$ wiedergegeben. Freies Eisen ist hochtoxisch, sodass eine Komplexbindung erforderlich ist. Die Präparate unterscheiden sich in der Kohlenhydrathülle und damit in der Stabilität, Sicherheit und Dosierung ( $\triangleright$ Tab. 3).

Eisenpräparate basierend auf hochmolekularem Dextran werden in Deutschland nicht mehr vertrieben, da sie gehäuft mit anaphylaktischen Reaktionen behaftet waren.

Niedermolekulares Eisendextran, z. B. Cosmofer ${ }^{\circledR}$, ist mit weniger unerwünschten Ereignissen assoziiert als hochmolekulares Eisendextran [24]. Der Hersteller empfiehlt intravenöse Infusionen von 100 bis $200 \mathrm{mg}$ Eisen pro Tag an 2 bis 3 Tagen pro Woche. Eine Gesamtdosisinfusion mit bis zu 20 mg Eisen pro Kilogramm Körpergewicht ist im Krankenhaus möglich, wobei detaillierte Informationen zu diesen Studien nicht zur Verfügung stehen [25]. 


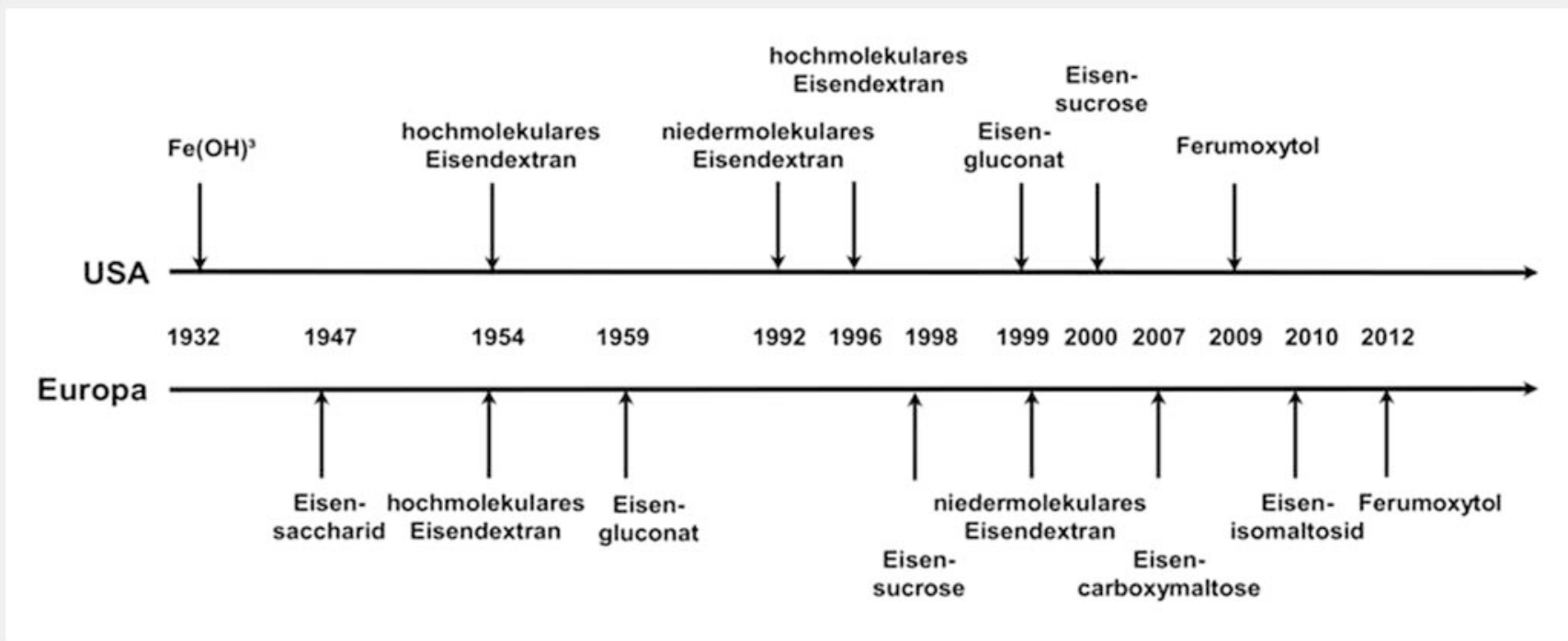

- Abb.1 Chronologie der Einführungen parenteraler Eisenformulierungen in Europa und den USA [23].

- Tab. 3 Eigenschaften von in Deutschland zugelassenen parenteralen Eisenpräparaten im Überblick.

\begin{tabular}{|c|c|c|c|c|c|}
\hline Freiname & $\begin{array}{l}\text { Niedermolekulares } \\
\text { Eisendextran }\end{array}$ & Eisengluconat & Eisensaccharose & Eisencarboxymaltose & Eisen-Derisomaltose \\
\hline Handelsname & Cosmofer & Ferrlecit & Venofer/FerMed & Ferinject & MonoFer \\
\hline Kohlenhydrathülle & $\begin{array}{l}\text { Dextran } \\
\text { (verzweigtes } \\
\text { Polysaccharid) }\end{array}$ & $\begin{array}{l}\text { Gluconat } \\
\text { (Monosaccharid) }\end{array}$ & $\begin{array}{l}\text { Sucrose } \\
\text { (Disaccharid) }\end{array}$ & $\begin{array}{l}\text { Carboxymaltose } \\
\text { (verzweigtes } \\
\text { Polysaccharid) }\end{array}$ & $\begin{array}{l}\text { Derisomaltose } \\
\text { (lineares } \\
\text { Oligosaccharid) }\end{array}$ \\
\hline Komplexstabilität & robust und stark & $\begin{array}{l}\text { labil und } \\
\text { schwach }\end{array}$ & $\begin{array}{l}\text { semirobust und } \\
\text { mittelstark }\end{array}$ & robust und stark & robust und stark \\
\hline $\begin{array}{l}\text { Molekulargewicht } \\
\text { (kDa) }\end{array}$ & 165 & 37,5 & $34-60 / 45$ & 150 & 150 \\
\hline $\begin{array}{l}\text { max. Eisendosis pro } \\
\text { Tag (mg) }\end{array}$ & $20 \mathrm{mg} / \mathrm{kg}$ & 62,5 & 500 & 1000 & $20 \mathrm{mg} / \mathrm{kg}$ \\
\hline $\begin{array}{l}\text { Gesamtdosisinfusion } \\
\text { (1 g bzw. } 20 \mathrm{mg} / \mathrm{kg} \text { ) }\end{array}$ & $\begin{array}{l}\text { ja (Krankenhausaufent- } \\
\text { halt erforderlich) }\end{array}$ & nein & nein & ja & ja \\
\hline $\begin{array}{l}\text { Verabreichungs- } \\
\text { geschwindigkeit }\end{array}$ & $\begin{array}{l}\text { zuerst } 25 \text { mg über } \\
15 \text { Minuten, danach bis zu } \\
150 \text { mg über } 30 \text { Minuten }\end{array}$ & $\begin{array}{l}\text { über } 20 \text { - } \\
30 \text { Minuten }\end{array}$ & $\begin{array}{l}7 \mathrm{mg} / \mathrm{kg} \mathrm{KG} \text { über } \\
31 \frac{1}{2} \text { Stunden }\end{array}$ & $\begin{array}{l}\text { Bolus: } 100 \text { mg/Min bis } \\
500 \text { mg Gesamtdosis; } \\
67 \text { mg/Min } 500 \text { mg bis } \\
1000 \text { mg Gesamtdosis }\end{array}$ & $\begin{array}{l}\text { Bolus: } 250 \mathrm{mg} / \text { Min } \\
\text { bis } 500 \mathrm{mg} \text { Gesamt- } \\
\text { dosis bis } 3 \mathrm{mal} \text { pro } \\
\text { Woche }\end{array}$ \\
\hline
\end{tabular}

Eisengluconat (Ferrlecit ${ }^{\circledR}$ ) und Eisensaccharose $\left(\right.$ Venofer ${ }^{\circledR}$, FerMed ${ }^{\circledR}$ ) waren die ersten intravenösen Eisenpräparate, die keine Dextranhülle aufweisen. Vergleichsstudien legten nahe, dass der pathophysiologische Unverträglichkeitsmechanismus bei niedriger Dosis von Eisengluconat nicht in einer Allergie, sondern in einer sogenannten „Pseudoallergie“ (anaphylaktoiden Reaktion) zu sehen ist [26]. Weil die Komplexe mit den Mono- bzw. Disaccharidhüllen nur moderat stabil sind, können nur begrenzte Mengen Eisen als Einzeldosis appliziert werden. Bei Eisengluconat beträgt die Maximaldosis pro Infusion $62,5 \mathrm{mg}$ Eisen [27]; bei Einzeldosen $\geq 125 \mathrm{mg}$ traten bei ca. jedem 20. Patienten Unverträglichkeitsreaktionen auf $[27,28]$. Bei Eisensaccharose ist seit kurzem die Gabe einer Einzeldosis von 500 mg zugelassen bei einer Infusionszeit von min. 3,5 Stunden [29].

Eisencarboxymaltose (Ferinject ${ }^{\circledR}$ ) und Eisenisomaltosid/EisenDerisomaltose (MonoFer ${ }^{\circledR}$ ) sind am höchsten dosierbar. Mit Eisencarboxymaltose sind Einzelgaben von bis zu $1000 \mathrm{mg}$ Eisen möglich. Bei Eisen-Derisomaltose beträgt die max. Dosierung $20 \mathrm{mg} / \mathrm{kg}$ Körpergewicht. Die Infusionen bzw. Injektionen erfolgen über Minuten bis max. eine Stunde ( $>$ Tab.3). Bei beiden Präparaten beträgt der zeitliche Abstand bis zum nächsten Termin mindestens eine Woche [30, 31].

Eine Strukturanalyse der verschiedenen Eisenpräparate zeigte sich in der Gelpermeationschromatografie für alle weiterhin 


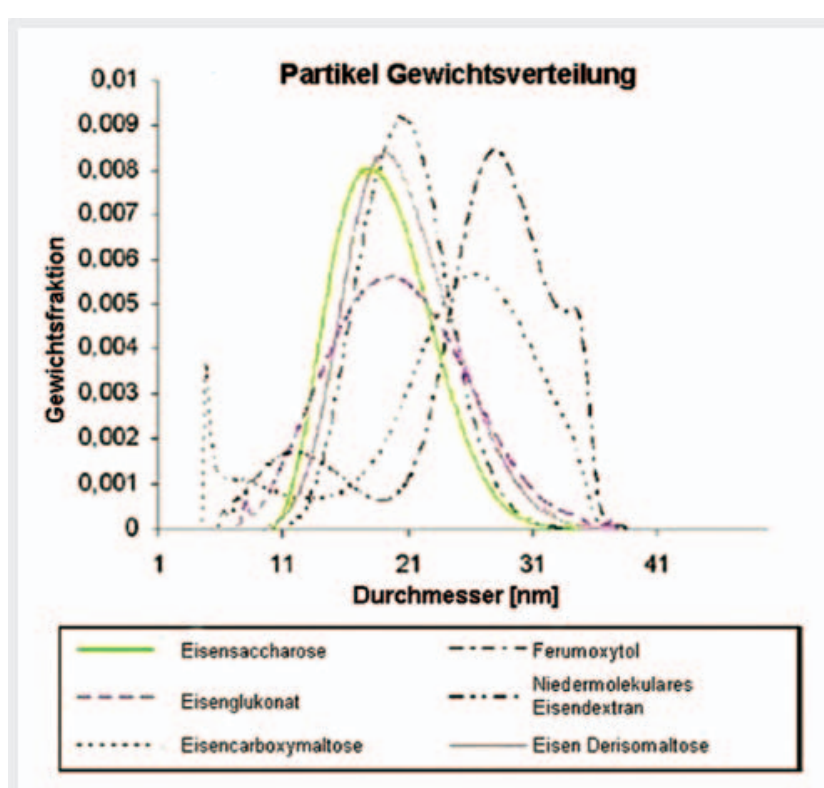

- Abb.2 Gewichtsverteilung vs. Partikeldurchmesser der intravenösen Eisenpräparate in der Gelpermeationschromatografie [32].

erhältlichen Präparate außer Eisencarboxymaltose und Ferumoxatol eine homogene Gewichtsverteilung in Abhängigkeit vom Durchmesser der Partikel. Eisencarboxymaltose zeigte einen weiteren Peak mit Partikeln größeren Durchmessers ( $\triangleright$ Abb. 2) [32].

Schließlich ergaben die Untersuchungen, dass der Gehalt an labilem und freiem Eisen in allen Präparaten, mit denen hohe Dosierungen möglich sind, gering war, wobei die neuesten Präparate am besten abschnitten ( $\mathbf{A} \mathbf{b} \mathbf{b} . \mathbf{3}$ ). Eisen-Derimaltose wird zunächst durch Hydrolyse von niedrig molekularem Dextran-1 hergestellt, jedoch wird es nach der chemischen Reduktion der Zuckerreste isoliert, um Redoxreaktionen oder Degradation der Aldehyd-Gruppe im anomerischen Zentrum zu verhindern. Die ${ }^{13} \mathrm{C}$ und ${ }^{1} \mathrm{H}$ NMR-spektroskopischen Analysen ergeben, dass Eisen-Derimaltose mit einem durchschnittlichen Molekulargewicht von 1000 Da keine verzweigten Strukturen aufweist [33]. Durch die chemischen Veränderungen ist das Eisen-Derisomaltose-Molekül nicht mehr mit bisherigen Dextranprodukten vergleichbar.

\section{Kritische Bewertung der Evidenz zur Eisengabe}

Niereninsuffizienz: Während bei Dialysepatienten im Rahmen einer randomisierten, kontrollierten Studie durch eine Anhebung erniedrigter Hb-Werte mittels ESAs eine Verbesserung der Lebensqualität dokumentiert wurde, steht dies bezogen auf eine Eisensubstitution für die CKD-Population noch aus. Unbestreitbar kann der Bedarf an ESAs durch Eisengabe reduziert werden.

Herzinsuffizienz: Die ESC-Leitlinien basieren im Wesentlichen auf zwei randomisierten, verblindeten Studien an Patienten mit Herzinsuffizienz bei reduzierter Auswurfsfraktion und Eisenmangel: Ferinject Assessment in Patients With Iron Deficiency and Chronic Heart Failure (FAIR-HF) $(n=459)$ [34] sowie Ferric Carb-

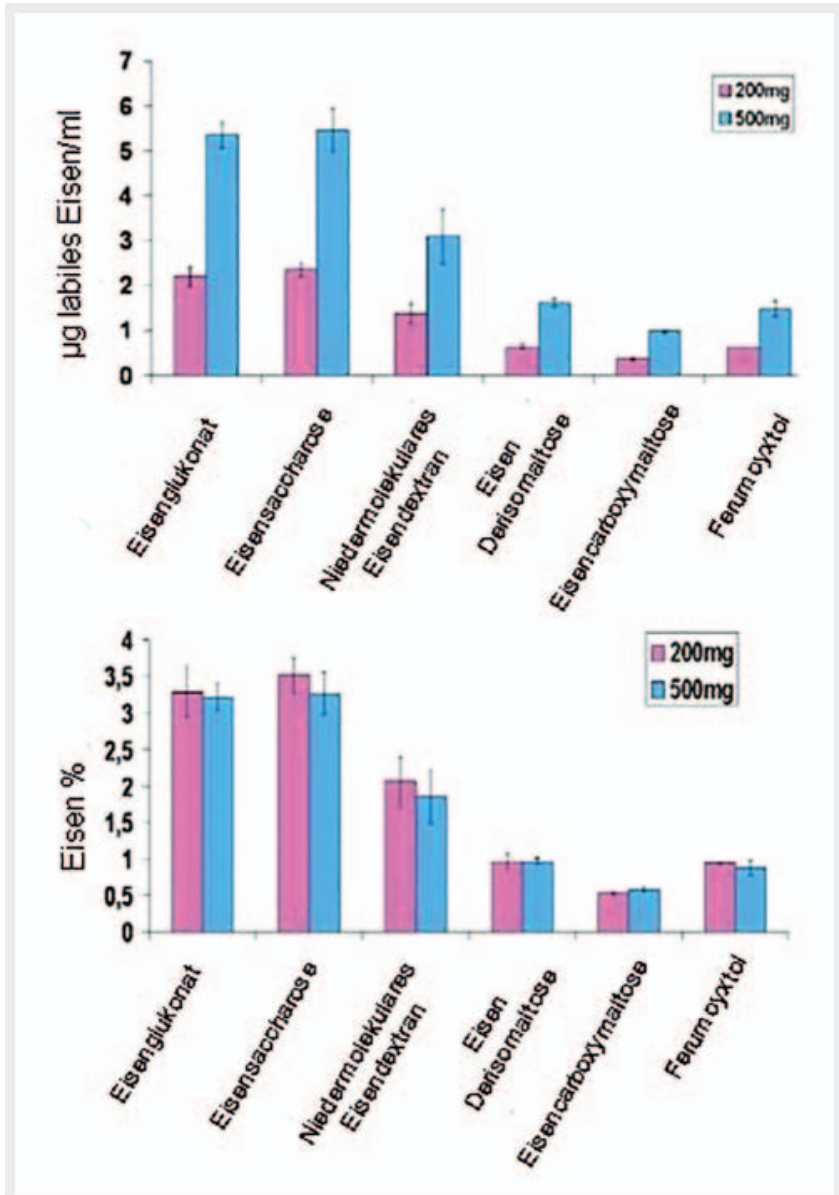

- Abb. 3 Gehalt an labilem Eisen der verschiedenen intravenösen Eisenpräparate im Vergleich. Oben: Konzentration des mittels Ferrozin nachweisbaren labilen Eisengehalts in $\mu \mathrm{g} / \mathrm{ml}$. Unten: Mittels Ferrozin nachweisbares labiles Eisen als Prozentsatz der eingesetzten Gesamtdosis [32].

oxymaltose Evaluation on Performance in Patients With Iron Deficiency In Combination With Chronic Heart Failure (CONFIRM-HF) ( $n=301$ ) [35]. In FAIR-HF und CONFIRM-HF stellten subjektives Befinden bzw. Leistungsfähigkeit sowie Hospitalisation die primären Endpunkte dar. Beide Studien ergaben eine Verbesserung der Lebensqualität unter Eisensubstitution. Während die Hospitalisation in CONFIRM-HF in der Eisengruppe statistisch gesenkt wurde, zeichnete sich in der FAIR-HF diesbezüglich ein Trend ab. Die FAIRStudie ergab eine Steigerung der Leistungsfähigkeit um $10 \%$ unter intravenösem Eisen; in CONFIRM-HF basierte die signifikante Leistungsdifferenz im Wesentlichen auf einer Verschlechterung in der Placebogruppe. Eine aktuelle Metaanalyse von Studien an 839 Probanden mit bis zu 52 Wochen Dauer ergab, dass die Hospitalisierung signifikant, aber die Mortalität nicht gesenkt wird [36]. Große kontrollierte Therapiestudien bei Herzinsuffizienten mit Mortalität als primärem Endpunkt rekrutieren gerade. Bemerkenswert ist, dass in CONFIRM-HF der positive Effekt von i. v. Eisen mit der Nierenfunktion signifikant korrelierte. Hingegen erbrachte eine Herzinsuffizienzstudie über 16 Wochen an $111 \mathrm{~Pa}-$ tienten mit täglich oralem Eisenpolysaccharid $(300 \mathrm{mg}$ ) versus 
114 Patienten mit Placebo keine Verbesserung der klinischen und echokardiografischen Parameter [37], wobei sich bezeichnenderweise auch keine Verbesserung der Eisen-laborchemischen sowie der hormonellen Werte abzeichnete. Auch diese Studie unterstreicht die Ineffizienz einer oralen Eisengabe. Die parenterale Eisengabe bei Patienten mit eingeschränkter systolischer linksventrikulärer Funktion wird in den neuesten US-amerikanischen kardiologischen Guidelines als intermediär positiv modulierend auf Komorbidität klassifiziert [38].

Chronische Darmerkrankungen: Bei Patienten mit CED (mit oder ohne Anämie) verbessert die Therapie des Eisenmangels die Lebensqualität. Hierbei wurden in Abhängigkeit vom Hämoglobin $(>/<10 \mathrm{~g} / \mathrm{d})$ und Körpergewicht $(>/<70 \mathrm{~kg})$ Eisendosen zwischen 1 und $2 \mathrm{~g}$ i. v. verabreicht [19].

Malignompatienten: Bei anämischen Krebspatienten vermindert sie den Bedarf an Bluttransfusionen. Hämoglobinanstiege von ca. 1,5-2,5 g/dl sind möglich. Allerdings konnte eine Cochrane-Analyse 2016 keine wesentliche Verbesserung der Lebensqualität zusätzlich zur ESA-Therapie zeigen [39]. Zwar ergab eine randomisierte US-Veterans-Administration-Studie über 4 1⁄2 Jahre bei 636 Patienten mit Aderlass vs. 641 Kontrollen, um kardiovaskuläre Ereignisse zu reduzieren, in der Eisenreduktionsgruppe ein niedrigeres Risiko neuer Malignome (38 vs. 60, Hazard Ratio $[H R]=0,65 ; P=0,036)$ sowie eine Reduktion der Mortalität bei Neuerkrankungen $(H R=0,49 ; P=0,009)$ [40]. Jedoch sind die Ergebnisse vieler anderer Studien hierzu uneinheitlich.

Nicht anämische Patienten: Das Befinden von nicht anämischen Frauen ohne weitere internistische Komorbiditäten nach intravenöser Eisengabe besserte sich, sofern das Ferritin anfangs $<15 \mathrm{ng} / \mathrm{ml}$ betrug [41].

\section{Sicherheit und zukünftige Entwicklungen}

Bei wiederholter Eiseninfusion muss das Thema einer potenziellen Akkumulation angesprochen werden: Mittels Kernspin-Technik kann die Eisenkonzentration in der Leber dargestellt werden, jedoch kann hiermit nicht zwischen parenchymatöser und retikuloendothelialer Deposition unterschieden werden, und nur die erste Form ist mit Toxizität assoziiert. Hinsichtlich der pathologischen Akkumulation von Eisen im Pankreas und Herz liegen bei Dialysepatienten kaum Studien vor.

Hypersensitivitätsreaktionen treten bei modernen i. v. Präparaten im Vergleich zu hochmolekularen Eisendextranen seltener auf. Eine Übersichtsarbeit gibt das Risiko für schwere unerwünschte Ereignisse mit weniger als einer pro 250000 Verabreichungen an [9]. Eine Analyse von knapp 690000 US-Versicherten aus den Jahren 2003 bis 2013 kam auf 68 Anaphylaxien/100 000 Applikationen bei nicht näher definierten Eisen-Dextran-Präparaten im Vergleich zu 24/100 000 bei Dextran-freien Präparaten [42], wobei es keine Definition für eine Anaphylaxie gab.

Eine Metaanalyse von 103 Studien mit über 19000 Patienten zur Sicherheit von i.v. Eisenpräparaten (10390 Patienten intravenös, 155 intramuskulär, 4044 oral, 1329 kein Eisen, 3335 Placebo) ergab, dass unter intravenösem Eisen nicht mehr schwerwiegende unerwünschte Ereignisse auftraten als unter den anderen, bzw. Nicht-Behandlungsformen. Zwar traten unter intravenösem
Eisen bis zu 2,5-mal mehr Infusionsreaktionen auf, jedoch wurden Anaphylaxien oder Todesfälle in keiner der eingeschlossenen Studien registriert. Ein erhöhtes Infektionsrisiko unter i.v. Eisen wurde in dieser Analyse ebenfalls nicht beobachtet. Gastrointestinale Nebenwirkungen und das Risiko für einen Therapieabbruch waren unter intravenösem Eisen vermindert [3].

Im Jahr 2013 erfolgte durch die EMA eine Überprüfung von i. v. Eisen, nachdem sich in Frankreich ein Risikosignal für schwere allergische Reaktionen bei Schwangeren gezeigt hatte [43]. Die EMA entschied, dass der Nutzen das Risiko überwiegt, sofern Maßnahmen zur Minimierung allergischer Reaktionen erfolgen. Bedingungen zur i. v. Eisenverabreichung (siehe Voraussetzungen in der Praxis).

\section{Voraussetzungen zur i. v. Verabreichung in der Praxis [4]}

Alle intravenösen Eisenpräparate sollten nur angewendet werden, wenn

- in der Erkennung und Behandlung anaphylaktischer Reaktionen geschulte Fachkräfte unverzüglich verfügbar sind und

- die kardio-pulmonale Reanimation durch eine entsprechende Ausrüstung sichergestellt ist.

Schließlich sollte die i. v. Anwendung in der Schwangerschaft auf das zweite und dritte Trimester begrenzt sein und nur erfolgen, wenn es unbedingt erforderlich ist.

Die EMA empfiehlt, die Patienten für mindestens 30 Minuten nach der Verabreichung hinsichtlich des Auftretens von Nebenwirkungen zu beobachten. Diese Vorgabe ist jedoch umstritten, denn bei einer intravenösen Eiseninfusion sind - anders als bei der subkutanen Antigenpräsentation nach einer Impfung oder Hyposensibilisierung - generell keine schweren verzögerten Reaktionen zu erwarten [44]. Ferner liegt keine Evidenz zur prophylaktischen Behandlung mit Kortikosteroiden oder Antihistaminika vor. Testdosen werden nicht mehr empfohlen, weil sie das Risiko für schwere Überempfindlichkeitsreaktionen nicht vorhersagen.

Zwar ergab eine retrospektive, internationale Beobachtungsstudie von > 30000 Hämodialysepatienten eine Assoziation zwischen erhöhten kumulativen i.v. Eisendosen und verschlechtertem Patienten-Outcome. Bei einer Gesamteisendosis von $\geq 300 \mathrm{mg} /$ Monat über 4 Monate zeigten sich eine signifikant höhere Mortalität und Hospitalisierungsrate im Vergleich zu niedrigeren monatlichen Gesamtdosen [45]. Jedoch können unkontrollierte Beobachtungsstudien keine Kausalitäten beweisen.

Zwei prospektive, randomisierte Vergleichsstudien oral vs. i. v. Eisen (REVOKE und FIND-CKD) ergaben sich widersprechende Ergebnisse [46, 47]. Während REVOKE bei i. v. Gabe bei nichtdialysepflichtigen CKD-Patienten eine höhere Rate an ernsthaften Ereignissen sowie Infektionen registrierte, sodass die Studie abgebrochen werden musste, ergaben sich bei FIND-CKD keine Gefahrensignale, weder für die i. v. Verabreichung noch für die Gruppe mit höherem Ferritinziel $(400-600 \mathrm{ng} / \mathrm{ml})$. In beiden Studien zeigte sich ein vergleichsweise verzögerter Anstieg des Hämoglo- 
bins unter oralem Eisen. Ergänzend hierzu ergab ein Vergleich Eisen-Derisomaltose vs. fraktionierte Eisensaccharose bei 1512 randomisierten anämischen Eisenmangelpatienten trotz Gleichheit der Gesamtdosen ein schnellerer Anstieg des Hämoglobins einhergehend mit einer rascheren Verbesserung des Müdigkeits-Scores unter Eisen-Derisomaltose [48].

Aktuell wurden im Herbst 2018 die Ergebnisse der mehrjährigen, randomisierten PIVOTAL-Studie veröffentlicht, die eine hochdosierte (d. h. $400 \mathrm{mg}$ im Monat i. v., solange die Ferritinkonzentration $<700 \mu \mathrm{g} / \mathrm{l}$ oder die TSAT $<40 \%$ betrug, im Median $264 \mathrm{mg} /$ Monat) mit einer minimalen (d. h. bis zu $400 \mathrm{mg}$ im Monat, falls Ferritin $<200 \mu \mathrm{g} / \mathrm{l}$ oder TSAT $<20 \%$, im Median $145 \mathrm{mg} /$ Monat) i.v. Eisentherapie mit Eisensaccharose bei insgesamt 2141 Hämodialysepatienten verglich [49]. Wie erwartet, wurden unter der höheren Eisendosis eine Abnahme der notwendigen EPO-Dosis sowie ein schnellerer Anstieg des Hb registriert. Die erhöhte Dosis erwies sich als sicher. Vermehrte Infektionen traten nicht auf. Die Gesamtmortalität war in der Hochdosisgruppe signifikant niedriger ( $R R$ 0,77; Cl 0,66-0,92). Bemerkenswert ist, dass in der Hochdosis-Gruppe weniger myokardiale Infarkte (RR 0,69; Cl 0,52-0,93) sowie weniger stationäre Aufenthalte wegen Herzinsuffizienz auftraten (RR 0,66; CI 0,46-0,94), womit letztendlich die Ergebnisse von FAIR und CONFIRM-HF auch in einer Dialyse-Kohorte bestätigt wurden.

Zusätzliche Daten zum i.v. Eisen sind in den kommenden Jahren zu erwarten, denn es werden noch weitere randomisierte Studien zur intravenösen Eisenbehandlung durchgeführt. Die Ironman-Studie mit ca. 1300 Teilnehmern im United Kingdom untersucht den Effekt von Eisen-Derisomaltose u.a. auf die kardiovaskuläre Mortalität, die Hospitalisierung sowie die Lebensqualität bei Patienten mit Herzinsuffizienz und Eisenmangel über einen Zeitraum von etwa 36 Monaten pro Patient [50]. Die in Deutschland durchgeführte FAIR-HF2 Studie vergleicht Eisencarboxymaltose mit Placebo und umfasst insgesamt 1200 Patienten mit Herzinsuffizienz über insgesamt 42 Wochen [51]. Die US-amerikanische HEART-FID-Studie analysiert bei 3014 Herzinsuffizienzpatienten Eisencarboxymaltose versus Placebo bezüglich u. a. Tod und Hospitalisierung über 12 Monate [52]. Die multinationale Affirm-AHF-Studie mit Eisencarboxymaltose gegen Placebo untersucht bei 1100 Patienten u. a. kardiovaskuläre Mortalität, Hospitalisierung und Lebensqualität über 12 Monate [53].

\section{Potenzielle Reduktion des Serum- phosphats}

Intravenöses Eisen (oder die Trägersubstanzen) interferiert mit dem Metabolismus von FGF23 - einem wichtigen Hormon zur Phosphatregulation [54]. Nach Eisencarboxymaltoseinfusion findet man eine Reduktion des Serumphosphats bis zu 3 Monaten [55]. Unter Eisensaccharosetherapie wird über das Auftreten einer hypophosphatämischen Osteomalazie spekuliert [56]. Das Risiko einer Hypophosphatämie ist möglicherweise kein Klasseneffekt aller intravenösen Eisenpräparate [57, 58]. Die klinische Bedeutung eines Abfalls des Serumphosphats bleibt beim Gros der Patienten zunächst spekulativ.

\section{KERNAUSSAGEN}

- Patienten mit Nieren- und/oder Herzinsuffizienz sowie chronisch-entzündlichen Darmerkrankungen und onkologische Patienten haben ein erhöhtes Risiko für einen Eisenmangel.

- Bei diesen Patientengruppen mit Anämie bietet die intravenöse Eisensubstitution subjektive, aber auch objektive Vorteile. Das Unverträglichkeitsrisiko ist gering.

- Es liegen mittlerweile die Ergebnisse 3 großer Studien vor, die insgesamt einen Benefit für einen wesentlichen Teil der Patienten belegen. Weitere Sicherheitsstudien mit harten Endpunktdaten werden für Herzkranke erwartet.

Interessenkonflikt

Patrick Biggar hat von Vifor-Fresenius Medical Care und Pharmacosmos Vortragshonorare erhalten. Vincent Brandenburg hat von Pharmacosmos Vortragshonorare erhalten. Die Abteilung von Peter Langguth hat Forschungsbeihilfen von Pharmacosmos, und Serumwerk Bernburg AG sowie eine Reisebeihilfe von Vifor erhalten.

Pierre-Alexandre Krayenbühl gibt keine Konfliktinteressen an.

Danksagung

Wir danken Herrn Professor Dr. med. K.U. Eckardt, Berlin, und Herrn Professor Dr. I.C. Macdougall, London, UK für ihre Unterstützung bei der Literatursuche.

Literatur

[1] Cappellini MD, Comin-Colet J, de Francisco A, IRON CORE Group et al. Iron deficiency across chronic inflammatory conditions: International expert opinion on definition, diagnosis, and management. Am J Hematol 2017; 92: $1068-1078$

[2] Mikhail A, Brown C, Williams JA, Kidney Disease: Improving Global Outcomes (KDIGO) Anemia Work Group et al. KDIGO Clinical Practice Guideline for Anemia in Chronic Kidney Disease. Kidney inter Suppl. 2012; 2: 279-335

[3] Avni T, Bieber A, Grossmann A et al. The safety of intravenous iron preparations: systematic review and meta-analysis. Mayo Clin Proc 2015; 90: $12-23$

[4] Arzneimittelkommission der deutschen Ärzteschaft. Verschärfte Empfehlungen bezüglich des Risikos schwerer Überempfindlichkeitsreaktionen auf Eisen-Präparate zur intravenösen Applikation (Oktober 2013). Im Internet: https://www.akdae.de/Arzneimittelsicherheit/RHB/Archiv/ 2013/20131021.pdf, Stand: 16.04.2018

[5] Musallam KM, Taher AT. Iron deficiency beyond erythropoiesis: should we be concerned? Curr Med Res Opin 2018; 34: 81-93

[6] von Haehling S, Gremmler U, Krumm M et al. Prevalence and clinical impact of iron deficiency and anaemia among outpatients with chronic heart failure: The PrEP Registry. Clin Res Cardiol 2017; 106: 436-443

[7] Ott C, Liebold A, Taskes A et al. High prevalence but insufficient treatment of iron-deficiency anemia in patients with inflammatory bowel disease: results of a population-based cohort. Gastroenterol Res Pract 2012; 2012: 595970

[8] Steinmetz HT. The role of intravenous iron in the treatment of anemia in cancer patients. Ther Adv Heamatol 2012; 3: 177-191 
[9] Muñoz M, Gomez-Ramirez S, Besser M et al. Current misconceptions in diagnosis and management of iron deficiency. Blood Transfus 2017; 15: $422-437$

[10] Nairz M, Theurl I, Wolf D et al. Iron deficiency or anaemia of inflammation? Wien Med Wochenschr 2016; 166: 411 -423

[11] Larson DS, Coyne DW. Understanding and exploiting hepcidin as an indicator of anemia due to chronic kidney disease. Kidney Res Clin Pract 2013; 32: $11-15$

[12] Gstrein C, Meyer M, Anabitarte P. Iron substitution in the treatment of chronic heart failure. Swiss Med Wkly 2017; 11: 100

[13] Ludwig H, Evstatiev R, Kornek G et al. Iron metabolism and iron supplementation in cancer patients. Wien Klin Wochenschr 2015; 127: 907 919

[14] Mei Z, Flores-Ayala RC, Grummer-Strawn LM et al. Is Erythrocyte Protoporphyrin a Better Single Screening Test for Iron Deficiency Compared to Hemoglobin or Mean Cell Volume in Children and Women? Nutrients 2017; 9: E557

[15] Patel D, Trivedi C, Khan N. Management of Anemia in Patients with Inflammatory Bowel Disease (IBD). Current Treat Options Gastro 2018; 16: $112-128$

[16] Okam MM, Koch TA, Tran MH. Iron Supplementation, Response in IronDeficiency Anemia: Analysis of Five Trials. Am J Med 2017; 130: 991

[17] Ganzoni AM. Eisen-Dextran intravenös: therapeutische und experimentelle Möglichkeiten. Schweiz Med Wochenschr 1970; 100: 301 - 303

[18] Tolkien Z, Stecher L, Mander A et al. Ferrous sulfate supplementation causes significant gastrointestinal side-effects in adults: a systematic review and meta-analysis. PLoS One 2015; 10: e0117383

[19] Shepshelovich D, Rozen-Zvi B, Avni T et al. Intravenous Versus Oral Iron Supplementation for the Treatment of Anemia in CKD: An Updated Systematic Review and Meta-analysis. Am J Kidney Dis 2016; 68: 677-690

[20] Ponikowski P, Voors AA, Anker SD et al. 2016 ESC Guidelines for the diagnosis and treatment of acute and chronic heart failure. Eur Heart ] 2016; 37: $2129-2200$

[21] Dignass AU, Gasche C, Bettenworth D et al. European consensus on the diagnosis and management of iron deficiency and anaemia in inflammatory bowel diseases. J Crohns Colitis 2015; 9: 211-222

[22] Aapro M, Beguin Y, Bokemeyer C, ESMO Guidelines Committee et al. Management of anaemia and iron deficiency in patients with cancer: ESMO Clinical Practice Guidelines. Ann Oncol 2018; 29 (Suppl. 4): iv96 iv110. doi:10.1093/annonc/mdx758

[23] Auerbach M, Ballard H. Clinical use of intravenous iron: administration, efficacy, and safety. Hematology Am Soc Hematol Educ Program 2010; 2010: $338-347$

[24] Auerbach M, Macdougall I. The available intravenous iron formulations: History, efficacy, and toxicology. Hemodial Int 2017; 21: S83-\$92

[25] Fachinformation Cosmofer $50 \mathrm{mg} / \mathrm{ml}$. Im Internet: https://s3.eu-central1.amazonaws.com/prod-cerebro-ifap/media_all/72143.pdf. Stand: Februar 2014

[26] Coyne DW, Adkinson NF, Nissenson AR et al. Sodium ferric gluconate complex in hemodialysis patients. II. Adverse reactions in iron dextransensitive and dextran-tolerant patients. Kidney Int 2003; 63: 217 - 224

[27] Sanofi. Fachinformation Ferrlecit $40 \mathrm{mg} /$ Ferrlecit $62,5 \mathrm{mg}$ ( ). Im Internet: https://mein.sanofi.de/produkte/Ferrlecit/Downloads? id=e730fd51-e5cf-41b4-9a09-7d4429fd8fd3. Stand: Juli 2017

[28] Michael B, Coyne DW, Folkert VW et al. Sodium ferric gluconate complex in haemodialysis patients: a prospective evaluation of long-term safety. Nephrol Dial Transplant 2004; 19: 1576-1580

[29] Fachinformation Fermed $20 \mathrm{mg} / \mathrm{ml}$. Im Internet: https://imedikament.de/ fermed-20-mg-ml/fachinformation. Stand: Oktober 2017

[30] Fachinformation MonoFer $100 \mathrm{mg} / \mathrm{ml}$. Im Internet: https://s3.eu-central1.amazonaws.com/prod-cerebro-ifap/media_all/49872.pdf. Stand: Juli 2017
[31] Fachinformation Ferinject $50 \mathrm{mg}$ Eisen/ml. Im Internet: https://s3.eucentral-1.amazonaws.com/prod-cerebro-ifap/media_all/76841.pdf. Stand: April 2017

[32] Jahn MR, Andreasen HB, Fütterer S et al. A comparative study of the physicochemical properties of iron isomaltoside 1000 (Monofer), a new intravenous iron preparation and its clinical implications. Eur J Pharm Biopharm 2011; 78: 480 - 491

[33] Kalra PA, Bock K, Meidal M. Iron isomaltoside 1000: a new high dose option for parenteral iron therapy. Port J Nephrol Hypert 2012; 26: 13 24

[34] Anker SD, Comin Colet J, Filippatos G et al. Ferric carboxymaltose in patients with heart failure and iron deficiency. N Engl J Med 2009; 361: $2436-2448$

[35] Ponikowski P, van Veldhuisen DJ, Comin-Colet J et al. Beneficial effects of long term intravenous iron therapy with ferric carboxymaltose in patients with symptomatic heart failure and iron deficiency. Eur Heart ] 2015; 36: $657-668$

[36] Anker SD, Kirwan BA, van Veldhuisen DJ et al. Effects of ferric carboxymaltose on hospitalisations and mortality rates in iron-deficient heart failure patients: an individual patient data meta-analysis. Eur J Heart Fail 2018; 20: 125-133

[37] Lewis GD, Malhotra R, Hernandez AF, NHLBI Heart Failure Clinical Research Network et al. Effect of Oral Iron Repletion on Exercise Capacity in Patients With Heart Failure With Reduced Ejection Fraction and Iron Deficiency: The IRONOUT HF Randomized Clinical Trial. JAMA 2017; 317: $1958-1966$

[38] Yancy CW, Januzzi JL Jr, Allen LA et al. 2017 ACC Expert Consensus Decision Pathway for Optimization of Heart Failure Treatment: Answers to 10 Pivotal Issues About Heart Failure With Reduced Ejection Fraction. JACC 2017; 72: $201-230$

[39] Mhaskar R, Wao H, Miladinovic B et al. The role of iron in the management of chemotherapy-induced anemia in cancer patients receiving erythropoiesis-stimulating agents. Cochrane Database Syst Rev 2016; 2: CD009624

[40] Zacharski LR, Chow BK, Howes PS et al. Decreased cancer risk after iron reduction in patients with peripheral arterial disease: results from a randomized trial. J Natl Cancer Inst 2008; 100: 996-1002

[41] KrayenbuehI PA, Battegay E, Breymann C et al. Intravenous iron for the treatment of fatigue in nonanemic, premenopausal women with low serum ferritin concentration. Blood 2011; 118: 3222 - 3227

[42] Wang C, Graham DJ, Kane RC et al. Comparative risk of anaphylactic reactions associated with intravenous iron products. JAMA 2015; 314: $2062-2068$

[43] Assessment report for: Iron containing intravenous (IV) medicinal products, 13 September 2013 EMA/549569/2013. Im Internet: http://www. ema.europa.eu/docs/en_GB/document_library/Referrals_document/ IV_iron_31 / WC500150771.pdf; Stand: 12.01.2018

[44] MacDougall IC, Bircher AJ, Eckardt KU et al. Conference Participants. Iron management in chronic kidney disease; conclusions from a "Kidney Disease: Improving Global Outcomes” (KDIGO) Controversies Conference. Kidney Int 2016; 89: 28 -39

[45] Bailie GR, Larkina M, Goodkin DA et al. Data from the Dialysis Outcomes and Practice Patterns Study validate an association between high intravenous iron doses and mortality. Kidney Int 2015; 87: 162 - 168

[46] Agarwal R, Kusek JW, Pappas MK. A randomized trial of intravenous and oral iron in chronic kidney disease. Kidney Int 2015; 88: 905 - 914

[47] Macdougall IC, Bock AH, Carrera F et al. FIND-CKD: a randomized trial of intravenous ferric carboxymaltose versus oral iron in patients with chronic kidney disease and iron deficiency anaemia. Nephrol Dial Transplant 2014; 29: 2075 - 2084

[48] Auerbach M, Lykke LL. A Single Infusion of Iron Isomaltoside 1000 Allows a More Rapid Hemoglobin Increment Than Multiple Doses of Iron Sucrose 
with a Similar Safety Profile in Patients with Iron Deficiency Anemia. Blood 2018; 132: 2334. doi:10.1182/blood-2018-99-110199

[49] Macdougall IC, White C, Anker SD et al. Intravenous Iron in Patients Undergoing Maintenance Hemodialysis. N Engl J Med 2018. (epub mit Update Januar 14, 2019)

[50] Kalra P. Intravenous Iron Treatment in Patients With Heart Failure and Iron Deficiency: IRONMAN. Im Internet: https://clinicaltrials.gov/ct2/ show/NCT02642562. Stand: 30.03.2018

[51] FAIR-HF-2 DZHK Trial 05 über Herzschwäche und Eisenmangel. Im Internet: https://fair-hf2.dzhk.de/ueber-die-studie/studiendesign/. Stand: 16.12.2018

[52] Randomized placebo-controlled trial if FCM as treatment for heart failure with iron deficiency (HEART-FID). Im Internet: https://clinicaltrials. gov/ct2/show/NCT03037931. Stand; 15.12.2018

[53] Study to compare Ferric Carboxymaltose with placebo in patients with acute heart failure and iron deficiency (Affirm-AHF). Im Internet: https:// clinicaltrials.gov/ct2/show/NCT02937454. Stand: 15.12.2018
[54] Muras-Szwedziak K, Nowicki M. Associations Between Intravenous Iron, Inflammation and FGF23 in Non-Dialysis Patients with Chronic Kidney Disease Stages 3-5. Kidney Blood Press Res 2018; 43: 143 -151

[55] Prats M, Font R, García C et al. Effect of ferric carboxymaltose on serum phosphate and C-terminal FGF23 levels in non-dialysis chronic kidney disease patients: post-hoc analysis of a prospective study. BMC Nephrol 2013; 14: 167

[56] Sato K, Nohtomi K, Demura H et al. Saccharated ferric oxide (SFO)-induced osteomalacia: in vitro inhibition by SFO of bone formation and 1,25-dihydroxy-vitamin D production in renal tubules. Bone 1997; 21 : $57-64$

[57] Bager P, Hvas CL, Dahlerup JF. Drug-specific hypophosphatemia and hypersensitivity reactions following different intravenous iron infusions. $\mathrm{Br}$ J Clin Pharmacol 2017; 83: 1118-1125

[58] Wolf M, Chertow GM, Macdougall IC et al. Randomized trial of intravenous iron-induced hypophosphatemia. JCI Insight 2018; 3: doi:10.1172 jci.insight. 124486 